\title{
Epidémiologie de la maladie de Newcastle en milieu rural au Mali
}

\author{
M. Sylla ${ }^{1}$ B. Traoré ${ }^{1}$ S. Sidibé ${ }^{2}$ \\ S. Keita ${ }^{1}$ F.C. Diallo ${ }^{1}$ B. Koné ${ }^{1}$ \\ A. Ballo ${ }^{1}$ M. Sangaré ${ }^{1}$ N'G. Koné ${ }^{1}$
}

Mots-clés

Volaille - Poulet - Pintade - Dindon Maladie de Newcastle - Mali.

\section{Résumé}

La maladie de Newcastle constitue une des principales contraintes au développement de l'aviculture villageoise au Mali. Les mortalités sont très élevées faute d'un plan de lutte adéquat. La présente étude, réalisée de 1996 à 2000, a eu pour but d'apporter une contribution à l'étude des particularités épidémiologiques de la maladie de Newcastle en milieu rural. Quatre sites ont été choisis dans les zones agroclimatiques soudanienne Sud, soudanienne Nord, sahélienne et du delta intérieur du Niger. Un observateur chargé du suivi clinicoépidémiologique dans les élevages a été basé dans chaque site. Dans chaque cas d'éclatement de foyer, des prélèvements ont été envoyés au Laboratoire central vétérinaire pour être analysés. L'étude a permis d'établir que le taux moyen de prévalence était de 32,9 p. 100 avec une variation entre 12,1 p. 100 en zone soudanienne Sud et 38,8 p. 100 en zone sahélienne. La saison sèche froide, avec 63 p. 100 des cas, a été la période favorable à l'éclosion des foyers, comparativement à la saison pluvieuse au cours de laquelle a été enregistré un taux de 15 p. 100. Les espèces aviaires affectées ont été le poulet, la pintade et le dindon. Les taux de morbidité, de mortalité et de létalité ont varié respectivement entre 8 et 100 p. 100, entre 5,7 et 82 p. 100, et entre 10 et 100 p. 100 . Trois périodes de pics ont été établies : janvier, mai et novembre. En 1998 a été enregistré le nombre le plus élevé de cas de la maladie.

\footnotetext{
1. Institut d'économie rurale, programme Volaille, BP 262, Bamako, Mali Tél. : 2243582 ; fax : 223775

E-mail : progvolaille.sotuba@ier.ml

2. Laboratoire central vétérinaire, BP 2295, Bamako, Mali

Tél. : 2243344 ; fax : 249809

E-mail : labovet@datatech.net.ml oudglcv@datatech.toolnet.org
}

\section{INTRODUCTION}

L'aviculture villageoise, renfermant 90 à 95 p. 100 du cheptel aviaire malien estimé à 22 millions de sujets $(6,11)$, est une activité importante du sous-secteur de l'élevage au Mali. Son développement est devenu un élément de stratégie de développement économique et social dans plusieurs pays en développement. Son essor contribue largement à lutter contre la pauvreté, à assurer la sécurité alimentaire et à promouvoir la femme rurale (15). Depuis la dévaluation du franc Cfa en janvier 1994, l'aviculture villageoise en zone soudano-sahélienne est devenue exportatrice. La fluidité du circuit, son faible coût logistique et la résistance aux maladies de la race locale en sont les atouts du système. Toutefois, il existe de nombreux facteurs limitants pour le développement de 
cet élevage traditionnel, dont la maladie de Newcastle qui occasionne de fortes mortalités. Cette mortalité systématique a d'ailleurs entrainé une démotivation et un désintérêt profond du paysan à l'égard de l'élevage de volailles lorsqu'il n'était pas informé de la possibilité de vacciner ses oiseaux (13).

La maladie est causée par un paramyxovirus affectant les volailles, et les oiseaux sauvages et domestiques. Elle est caractérisée par une grande variabilité de la morbidité, de la mortalité, des signes cliniques et des lésions (12). Les poules, pintades, perdrix, faisans, cailles et dindes sont les espèces les plus sensibles (10). La présente étude, menée de 1996 à 2000, a eu pour but d'apporter une contribution à l'étude des particularités épidémiologiques de la maladie de Newcastle en milieu rural du Mali et de proposer un programme approprié de lutte.

\section{MATERIEL ET METHODES}

\section{Sites de l'étude}

Le choix des sites a été réalisé en collaboration avec les services techniques d'encadrement en raison de leur connaissance du milieu. Au moins trois localités, pouvant servir de sites potentiels, ont été proposées aux auteurs et un groupe de contacts a été organisé avec les aviculteurs dans chacun d'eux. Des informations sur les données générales du village et l'aviculture en particulier ont été récoltées. L'accessibilité des sites en toute saison, l'importance des effectifs aviaires, la présence d'un marché à volailles, d'associations d'aviculteurs, d'une chaîne de froid pour la conservation des prélèvements, l'incidence des maladies aviaires, l'adhésion du promoteur, ont été les critères qui ont permis d'opérer un choix définitif. Les sites retenus ont été Balamoussala en zone sahélienne, Tiendo en zone soudanienne Nord, Kignan en zone soudanienne Sud, Barbé dans le delta du Niger (figure 1) Les caractéristiques météorologiques des sites durant la période d'étude figurent au tableau I.

\section{Mode d'élevage}

L'aviculture villageoise est avant tout familiale, en petits effectifs individuels très dispersés, d'une productivité faible. Toutefois, elle est adaptée, avec des coûts d'intrants et d'investissements faibles, aux conditions de vie des populations paysannes, donc à leurs besoins alimentaires, sociaux et économiques. Plusieurs espèces et catégories physiologiques partagent le même habitat. Le poulet, avec plus de 80 p. 100 des effectifs, est l'espèce la plus représentative.

Les poulaillers sont en général de type traditionnel ou amélioré. Les normes de densité, d'aération et d'hygiène sont rarement respectées.

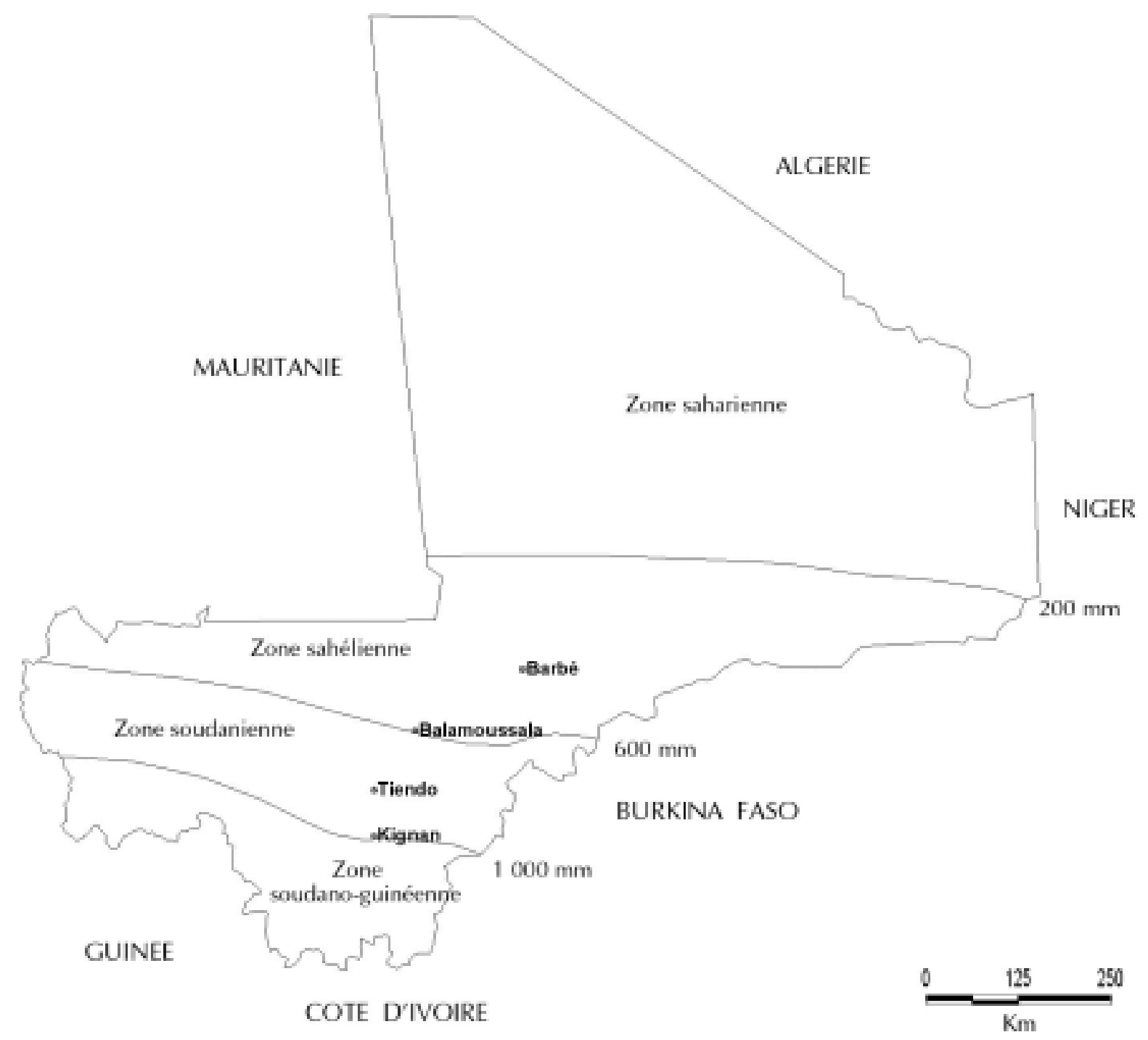

Figure 1 : zones agroclimatiques du Mali. 
Tableau I

Caractéristiques météorologiques des sites de l'étude : moyennes de 1996 à 2000

\begin{tabular}{|c|c|c|c|c|c|c|c|c|}
\hline Sites & $\begin{array}{l}\text { Altitude } \\
\qquad(\mathrm{m})\end{array}$ & $\begin{array}{c}\text { Longitude } \mathbf{O} \\
\left(^{\circ}\right)\end{array}$ & $\begin{array}{c}\text { Latitude } N \\
\left({ }^{\circ}\right)\end{array}$ & $\begin{array}{c}\left.\text { T. } \min .{ }^{1}{ }^{\circ} \mathrm{C}\right)\end{array}$ & $\begin{array}{c}\text { T. } \max .{ }^{2} \\
\left({ }^{\circ} \mathrm{C}\right)\end{array}$ & $\begin{array}{l}\text { H. rel. min. } \\
(\%)\end{array}$ & $\begin{array}{l}\text { H. rel. max. } \\
(\%)\end{array}$ & $\begin{array}{c}\text { Pluviométrie } \\
\text { (mm) }\end{array}$ \\
\hline Balamoussala & 288 & $-06,09$ & 13,24 & 22,7 & 36,7 & 37 & 69 & 662,1 \\
\hline Tiendo & 315 & $-06,48$ & 12,29 & 21,7 & 34,3 & 33 & 69 & 916,6 \\
\hline Kignan & 348 & $-06,01$ & 11,51 & 21,6 & 33,5 & 36 & 72 & 871,6 \\
\hline Barbé & 271 & $-04,06$ & 14,41 & 22,6 & 36,3 & 24 & 59 & 489 \\
\hline \multicolumn{9}{|c|}{${ }^{1}$ Température minimale } \\
\hline \multicolumn{9}{|c|}{${ }^{2}$ Température maximale } \\
\hline \multicolumn{9}{|c|}{${ }^{3}$ Humidité relative minimale } \\
\hline \multicolumn{9}{|c|}{${ }^{4}$ Humidité relative maximale } \\
\hline
\end{tabular}

Les mangeoires sont constituées de vieilles tôles, de bois ou de vieilles assiettes, et les abreuvoirs de boîtes de conserves ou de poteries en terre cuite. Les oiseaux glanent leur nourriture dans la nature. Un complément, constitué généralement de graines et/ou de son de céréales, est offert le matin à leur sortie du poulailler ou le soir à leur retour, suivant les moyens du paysan. Certains offrent aussi des termites à partir de termitières récoltées dans la nature.

\section{Suivi clinicoépidémiologique des élevages avicoles et prélèvements d'échantillons}

Un observateur, chargé du suivi clinicoépidémiologique dans les élevages, a été installé dans chaque site. Les aviculteurs ont été sensibilisés par rapport aux objectifs de l'étude et à la collaboration avec l'observateur qui devait effectuer un passage quotidien dans les élevages. Les observations ont porté sur l'évolution des effectifs, le nombre de sujets malades, la description des signes cliniques, le nombre de sujets morts, la période d'apparition des maladies, les espèces et catégories aviaires affectées.

Le suivi clinique a été fait tout au long de l'année, laquelle peut être subdivisée en saison sèche froide (de novembre à février), en saison sèche chaude (de mars à mai) et en saison pluvieuse (de juin à octobre). En cas de foyer, des prélèvements sanguins étaient effectués sur des oiseaux malades pour l'obtention de sérum. Après les autopsies sur des cadavres frais ou sacrifiés, des organes (poumons, trachée, larynx, cerveau, proventricule, gésier, ovaires, cloaque et caeca) étaient prélevés et envoyés au Laboratoire central vétérinaire de Bamako pour réaliser des examens.

\section{Techniques de diagnostic au laboratoire}

La confirmation des différentes suspicions de maladie de Newcastle a été basée essentiellement sur l'isolement du virus sur œufs embryonnés âgés de 9 à 11 jours et son identification a été faite à l'aide du test d'hémagglutination et d'inhibition de l'hémagglutination. Certains cas de suspicion de la maladie de Newcastle, chez des sujets n'ayant jamais été vaccinés, ont été confirmés à travers le dépistage d'anticorps spécifiques postinfectieux à l'aide du test d'inhibition de l'hémagglutination.

\section{Prévalence, taux de morbidité, de mortalité, de létalité et répartition saisonnière de la maladie}

Le taux de prévalence a été calculé en rapportant le nombre de cas confirmés sur le nombre total de cas suspects de maladie de
Newcastle. Les taux de morbidité, de mortalité et de létalité ont également été déterminés. Le taux de morbidité a été égal au nombre de sujets malades sur l'effectif total du cheptel de l'exploitation ; le taux de mortalité a été égal au nombre de sujets morts sur l'effectif total du cheptel de l'exploitation ; le taux de létalité a été égal au nombre de sujets morts sur le nombre de sujets malades dans l'exploitation. La répartition saisonnière a été déterminée en faisant le ratio du nombre de cas enregistrés au cours d'une saison donnée sur le nombre total de cas enregistrés dans l'année.

\section{RESULTATS}

\section{Signes cliniques}

La maladie a été caractérisée par la torpeur, l'abattement, la cyanose de la crête. Les troubles digestifs se sont manifestés le plus souvent par de l'anorexie et des diarrhées, et les troubles respiratoires par des halètements, de la dyspnée, des râles, du jetage bucco-nasale, de la toux. L'incoordination motrice, les tremblements, le torticolis, la paralysie des ailes et des pattes ont caractérisé les troubles nerveux. Une forme atypique, avec l'absence de signes nerveux et avec des signes respiratoires moins développés, a été également observée.

\section{Lésions}

Les principales lésions chez les sujets abattus ou morts ont été des hémorragies sous forme de pétéchies sur la muqueuse du proventricule, du gésier et de la paroi intestinale, l'accumulation de sécrétions visqueuses dans la cavité buccale et la trachée artère, une laryngite et une trachéite congestives.

\section{Caractéristiques épidémiologiques}

Les effectifs moyens de volailles ont varié par exploitation entre 29 sujets à Barbé et 71 à Tiendo. Les effectifs minimums ont varié entre 3 et 27 et les maximums entre 70 et 200 par exploitation. La maladie a été caractérisée par une haute contagiosité et a sévi sur les individus de tout âge. Sa propagation a été rapide du fait du contact permanent entre les volailles d'un même village et du transport de poulets entre villages. La contamination des élevages se fait par voie aérienne et explique la très grande transmission d'un élevage à l'autre de la maladie en période de vents.

Les espèces aviaires affectées ont été le poulet, la pintade et le dindon, de toutes catégories physiologiques. Au total, 104 foyers ont 
été enregistrés dans les quatre zones agroclimatiques. Le taux de prévalence par site est illustré à la figure 2 . Le taux de morbidité a varié entre 8 et 100 p. 100 , le taux de mortalité entre 5,7 et 82 p. 100 et le taux de létalité entre 10 et 100 p. 100.

La maladie a sévi principalement en saison sèche froide (63 p. 100), puis en saison sèche chaude (22 p. 100) et en saison pluvieuse (15 p. 100) (figure 3). Le taux de prévalence a augmenté progressivement de 1996 (18,8 p. 100) à 1998 (49,4 p. 100) pour décroître en 1999/2000 (figure 4). L'évolution annuelle de la maladie a montré trois pics : aux mois de janvier, mai et novembre (figure 5).

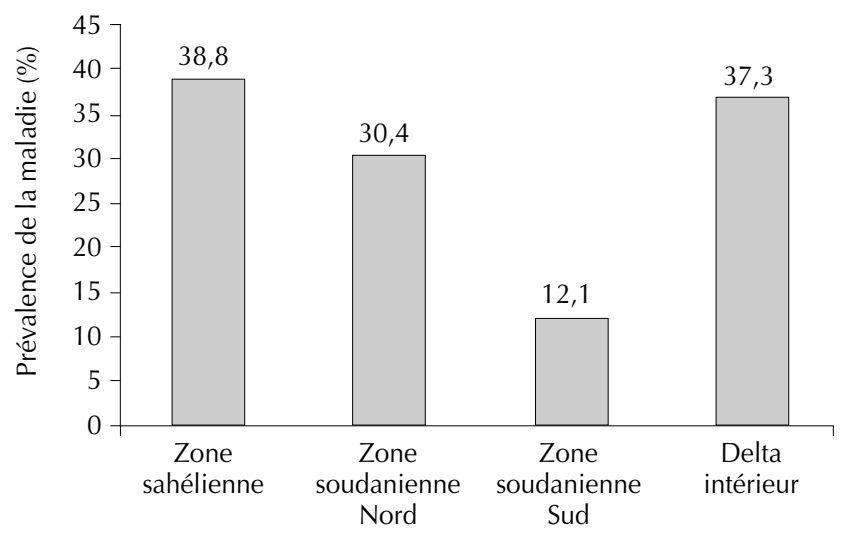

Figure 2 : taux de prévalence de la maladie de Newcastle par zone agroclimatique au Mali.

Saison sèche chaude

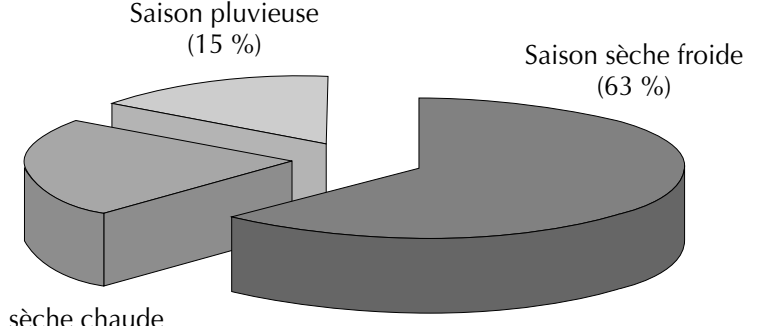
$(22 \%)$

Figure 3 : répartition saisonnière de la maladie de Newcastle au Mali.

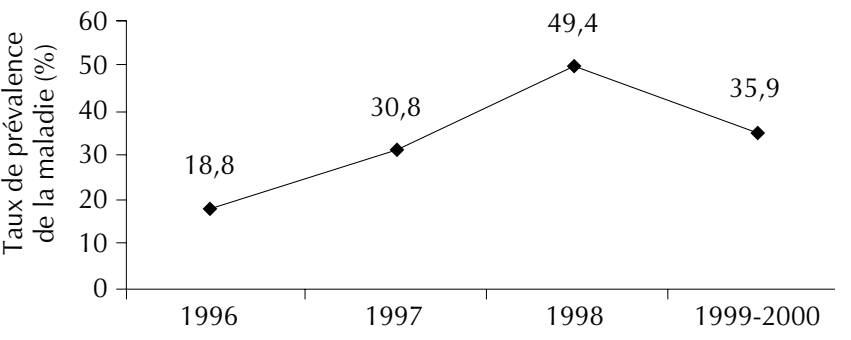

Figure 4 : variation pluriannuelle du taux de prévalence de la maladie de Newcastle au Mali.

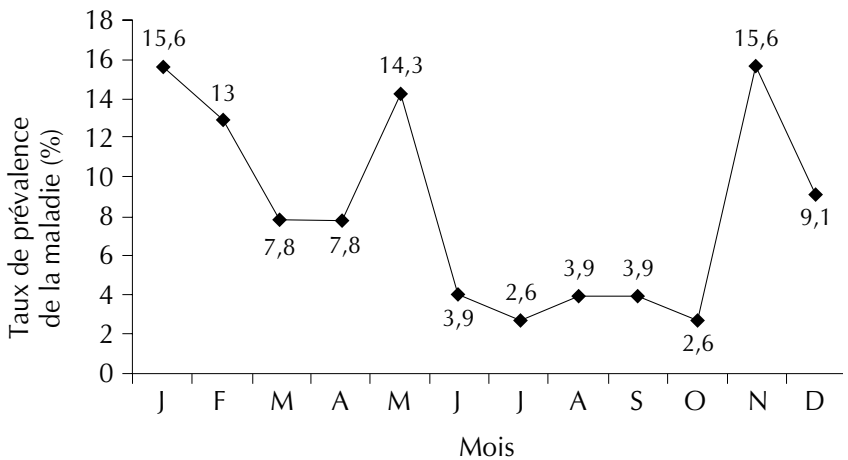

Figure 5 : évolution annuelle du taux de prévalence de la maladie de Newcastle au Mali.

\section{DISCUSSION}

Excepté le site de Kignan, le taux de prévalence a été supérieur à 30 p. 100 dans les autres sites. Dans ce site, la faible incidence de la maladie pourrait s'expliquer par l'implantation, durant la période d'étude, d'un important réseau de distribution des produits vétérinaires dans le Mali Sud, dont le vaccin contre la maladie de Newcastle, et la formation d'un nombre important de promoteurs d'élevages villageois grâce à l'appui du Projet d'appui à l'élevage Mali Sud III. Ainsi, entre 1995 et 1997, le chiffre de vaccination est passée de 100000 à plus de 3,5 millions par an dans ladite zone (13).

$\mathrm{Au}$ cours des enquêtes sérologiques sur les principales viroses de la volaille, entre 1989 et 1991, il est ressorti que le virus de la maladie de Newcastle circulait dans les cinq sites visités avec une prévalence variant entre 14,28 p. 100 en zone sahélienne et 59,09 p. 100 en zone soudanienne Nord (14). Contrairement à ces résultats d'enquêtes, c'est en zone sahélienne qu'a été enregistré le taux de prévalence le plus élevé (38,8 p. 100), tandis qu'en zone soudanienne Nord, il a été de 30,4 p. 100.

Les taux obtenus au cours de l'étude restent en deçà du taux de 70 p. 100 enregistré au cours d'enquêtes effectuées entre 1984 et 1992 (7) et des taux d'infection variant entre 46,6 p. 100 et 52,27 p. 100, suite à l'enquête menée dans les régions de Kayes, Koulikoro et le district de Bamako (8). En effet, les tailles d'échantillons dans les sites de la présente étude ont été nettement supérieures à celles des précédentes enquêtes $(7,8)$. Il en est de même pour celles rapportées au cours d'une enquête préliminaire sur la production des animaux de basse-cour dans la zone de Niono. Là, les effectifs moyens de poulets par famille dans la zone concernée étaient de 17,6 pour le système urbain, 21,6 pour le système riz et 11,4 pour le système mil (9).

Au Niger un taux de 14 p. 100 a été obtenu au cours d'une enquête sérologique réalisée sur les pathologies aviaires en milieu rural (4) et des taux variant entre 80 et 100 p. 100 ont été rapportés en Côte d'Ivoire (3). Les taux de mortalité enregistrés ont été comparables à ceux rapportés au Sénégal qui ont varié entre 30 et 70 p. 100, suite à une épidémie ayant affecté les exploitations avicoles de la zone de Dakar, entre décembre 1994 et juillet 1995 (1). La prévalence individuelle de la maladie de Newcastle en élevage traditionnel augmente significativement en saison sèche $(84 \mathrm{p}$. 100) et en saison des pluies (98 p. 100) (1).

Des études menées au Nigeria (2) et au Zaïre (5) ont montré des résultats similaires en fonction des saisons. Selon Bahus (2), c'est 
pendant la saison sèche (de janvier à mars) que la maladie est particulièrement virulente. D'après Eyanga (5), la maladie sévit dans la partie sud du Zaïre en janvier, février, mars et avril, contrairement à la partie nord où elle cause des ravages chez les oiseaux entre septembre et février. L'harmattan favoriserait la propagation du virus (3). Une recrudescence de la maladie a été constatée après 1999, correspondant à l'arrêt de certaines opérations de développement qui intervenaient dans la vaccination contre la maladie de Newcastle.

\section{CONCLUSION}

Le virus de la maladie de Newcastle circule dans les quatre zones agroclimatiques en toute saison. Les foyers épizootiques ont été observés en saison sèche froide et en saison sèche chaude. Les pertes causées ont été importantes. Toutes les espèces et catégories aviaires ont été affectées. Le taux de prévalence a varié d'une année à l'autre et le plus élevé a été enregistré en 1998. Les mois de janvier, mai et novembre ont été les trois périodes de pic de l'année.

Les volailles doivent être immunisées au plus tard en octobre pour assurer une protection durant la saison sèche froide et au plus tard en mars pour couvrir le reste de l'année. Les poussins nés après les campagnes de vaccination doivent être immunisés au fur et à mesure. Les immunisations doivent concerner les poulets mais aussi les autres espèces aviaires qui, sans être aussi sensibles que les poulets au virus de la maladie de Newcastle, constituent un maillon important dans le cycle épidémiologique de cette maladie. La vaccination, aussi efficace soit-elle, ne pourra agir pleinement que si elle est accompagnée de mesures sanitaires de prévention qui permettent soit d'éviter l'introduction du virus dans une région saine, soit de le faire disparaître à partir des foyers lors de l'apparition de la maladie.

\section{Remerciements}

Nous remercions sincèrement le Programme national de la recherche agricole qui a financé l'étude, toutes les structures locales de développement des sites qui ont bien voulu coopérer avec l'équipe de recherche, les observateurs pour leur courage et leur abnégation, les aviculteurs collaborateurs qui ont bien voulu mettre leur cheptel à notre disposition pendant toute la durée de l'étude et les agents d'appui technique, à tous les niveaux, sans lesquels ces résultats ne seraient disponibles.

\section{BIBLIOGRAPHIE}

1. ARBELOT B., DAYON J.F., MAMIS D., GUEYE J.C., TALL F., SAMB H., 1997. Enquête sérologique sur la prévalence des principales pathologies aviaires au Sénégal. Rapport. Dakar, Sénégal, Isra, Laboratoire de pathologies aviaires, $9 \mathrm{p}$

2. BAHUS J., 1993. La maladie de Newcastle aux premières loges. Afr. Agric., $\mathrm{n}^{\circ} 200$ : 15-16.

3. COUACY-HYMANN E., SANOGO B., DOMENECH J., 1991. Épidémiologie de la maladie de Newcastle en Côte d'Ivoire. In : Proc. Workshop Newcastle Disease Vaccines for Rural Africa, PANVAC, Debre Zeit, Addis Ababa, Ethiopia, 22-26 April 1991, p. 65-68.

4. COURTECUISSE C., JAPIOT F., BLOCH N., DIALLO I., 1990. Enquête sérologique sur la maladie de Newcastle et de Gumboro, la pasteurellose et la pullorose chez les poules de race locale au Niger. Revue Elev. Méd. vét. Pays trop., 43 : 27-29.

5. EYANGA E., 1991. La maladie de Newcastle au Zaïre. In : Proc. Workshop Newcastle Disease Vaccines for Rural Africa, PANVAC, Debre Zeit, Addis Ababa, Ethiopia, 22-26 April 1991, p. 69-72.

6. KOUNTA A.O.S., 1992. Note technique sur le développement de I'aviculture au Mali. Tropicultura, 10 : 103-105

7. KOUNTA A.O.S., 1993. Mali. Vers un développement de l'aviculture villageoise. Afric. Agric., $\mathrm{n}^{\circ} 202$ : 17-19.

8. KOUNTA A.O.S., 1995. Maladie de Newcastle. Principal fléau des élevages villageois. Afric. Agric., n $223: 56$.
9. KUIT H.G., TRAORE A., WILSON R.T., 1985. Enquête préliminaire sur les animaux de basse-cour dans la zone de Niono. Bamako, Mali, CIPEA. (Document de programme $n^{\circ}$ AZ 144A)

10. Maladie de Newcastle. Tout savoir sur le virus et la vaccination, 1996. Afr. Agric., $\mathrm{n}^{\circ} 234$ : 46-48.

11. Ministère de l'Agriculture, de l'élevage et des eaux et forêts, 1991. Rapport annuel. Bamako, Mali, direction nationale de I'Elevage.

12. MEULEMANS G., 1992. Maladie de Newcastle et infections à paramyxovirus. In : Manuel de pathologie aviaire. Maisons-Alfort, France, école nationale vétérinaire, 381 p.

13. REMOND G., FERMET QUINET E., 2000. La vaccination contre la maladie de Newcastle en aviculture villageoise. Un facteur de développement impliquant tous les acteurs de la filière. Afr. Agric., $n^{\circ} 281: 74-75$

14. TOUNKARA K., DIALLO B.O., SECK B.M., TRAORE A., SAMAKE K. 1995. Prévalence sérologique des principales viroses du bétail et de la volaille au Mali. Revue malienne Sci. Tech., $\mathrm{n}^{\circ} 3$ : 30-35.

15. TRAORE A., 1999. Requirements for family poultry development. First INFPD/FAO Electronic Conference on Family Poultry, the Scope and Effect of Family Poultry Research and Development.

Accepté le 29.03.2004 


\section{Summary}

Sylla M., Traoré B., Sidibé S., Keita S., Diallo F.C., Koné B., Ballo A., Sangaré M., Koné N'G. Epidemiology of Newcastle Disease in Rural Areas of Mali

The Newcastle disease constitutes one of the main constraints to poultry farming development at the village level in Mali, causing high mortality. This study was carried out between 1996 and 2000 with the aim to contribute to the epidemiological characteristics of the disease in rural areas of Mali and to propose a suitable plan for prevention. The four study sites were selected in the agroclimatic regions of Mali: North Sudanian, South Sudanian, Sahelian and Niger River inland delta. An observer in charge of monitoring the clinical and epidemiological aspects of the disease was based on each site. For each outbreak, samples were collected and sent to the Central Veterinary Laboratory of Bamako for analysis. The mean prevalence rate was $32.9 \%$ with a variation between $12.1 \%$ in the South Sudanian zone and $38.8 \%$ in the Sahelian zone. The majority of the outbreaks $(63 \%)$ occurred in the cold dry season, as opposed to the rainy season during which $15 \%$ of the cases were recorded. The main avian species affected were the chicken, fowl and turkey. Morbidity rates varied between $8-100 \%$, mortality rates between $5.7-82 \%$, and lethality rates between $10-100 \%$. The three peaks of the disease occurred in January, May and November. The highest number of Newcastle disease cases were recorded in 1998.

Key words: Poultry - Chicken - Guinea fowl - Turkey Newcastle disease - Mali.

\section{Resumen}

Sylla M., Traoré B., Sidibé S., Keita S., Diallo F.C., Koné B., Ballo A., Sangaré M., Koné N'G. Epidemiología de la enfermedad de Newcastle en un medio rural de Mali

La enfermedad de Newcastle constituye uno de los principales obstáculos al desarrollo de la avicultura de pueblo en Mali. Las mortalidades son muy elevadas, por falta de un plan de lucha adecuado. El presente estudio, realizado de 1996 a 2000, tuvo por finalidad la de aportar una contribución al estudio de las particularidades epidemiológicas de la enfermedad de Newcastle en medio rural. Se escogieron cuatro sitios en las zonas agro-climáticas sudanesa sur, sudanesa norte, sahariana y del delta interior del Níger. Se basó un observador encargado del seguimiento clínicoepidemiológico en cada producción. En cada caso de brote, se enviaron muestras para análisis al Laboratorio central veterinario. El estudio permitió establecer que la tasa media de prevalencia fue de $32,9 \%$, con una variación entre $12,1 \%$ en zona sudanesa sur y $38,8 \%$ en zona sahariana. La estación seca y fría, con $63 \%$ de los casos, fue el periodo favorable para la aparición de los brotes, en comparación con la estación lluviosa, durante la cual se registró una tasa de 15\%. Las especies avícolas afectadas fueron el pollo, la pintada (gallina de Guinea) y el pavo. Las tasas de morbilidad, de mortalidad y de letalidad variaron respectivamente entre $8 \%$ y $100 \%$, entre 5,7 y $82 \%$ y entre $10 \%$ y $100 \%$. Se establecieron tres periodos de picos: enero, mayo y noviembre. En 1998 se registró el número de casos más elevado.

Palabras clave: Ave de corral - Pollo - Gallina de Guinea Pavo - Enfermedad de Newcastle - Malí. 